\title{
The Memory Glasses: Subliminal vs.Overt Memory Support with Imperfect Information
}

\author{
Richard W. DeVaul, Alex "Sandy" Pentland \\ Massachusetts Institute of Technology \\ Media Laboratory \\ $\{$ rich, sandy $\}$ omedia.mit.edu
}

\author{
Vicka R. Corey \\ Harvard Medical School \\ A. A. Martinos Center for Biomedical Imaging \\ vicka@nmr.mgh.harvard.edu
}

\begin{abstract}
Wearables are frequently designed to support users engaged in complex "real world" activities, ranging from food inspection to ground combat. Unfortunately, wearables also have the potential to interfere with the very tasks they are designed to support, either by distracting the user or providing them with misleading information.

In 2002 we published a pilot study suggesting that a subliminal visual cuing system might be an effective lowattention interaction strategy for just-in-time memory support. In this paper we present the results of a larger study demonstrating that not only is wearable subliminal cuing significantly effective (increasing performance by a factor of approximately $1.5, p=0.02$ ), but even incorrect subliminal cues can actually improve performance. By contrast, consciously-visible incorrect cues caused performance to degrade.
\end{abstract}

\section{Introduction}

An HCI designer expecting a desktop environment can assume that the user is probably sitting down someplace safe, with few extraneous stimuli competing for their attention. By contrast, interaction design in the wearablecomputing domain must take far more into account. The user may be driving a car or engaging in combat, and such other tasks will divide their attention and limit their resources for interacting with the computer.

Wearable computing applications are frequently intended to provide support for complex real-world activities, from food inspection[14] to service and maintenance[24] to military command and control (SAAB WISE project, US Army Objective Force Warrior program). However, the use of such wearable task-support applications can interfere with the performance of the very task they are designed to support. They can be distracting, taking too much of the user's attention away from the task at hand. Or they can actively misdirect the user, giving wrong information or bad advice.

The risk of misleading the user is one task-support application designers should not ignore. Real-world environments are complex, and the information available to wearable task-support applications is likely to be limited and imperfect.

Unsurprisingly, our research suggests that presenting the user with incorrect information can result in a decrease in task performance. However, in at least some cases this effect may actually be inverted - improving user performance even when the computer's cue is incorrect - if the cue is presented subliminally, rather than consciously visible. (We discuss this surprising result further in Section 4.7.1 below.)

More complex than the problem of misdirection is the problem of divided attention. Contemporary theories of attention [4, 25] are based on finite resources of perception and cognition. Simultaneous tasks that require conscious attention compete for a person's limited attentive resources. The tasks interfere with one another, and the person's performance suffers on all of the tasks.

Interference issues are particularly problematic for wearable task-support applications. As the complexity of the real-world task increases, so does the need for task support. But at the same time, the user's capacity for attending to a task-support application decreases. Effective support is hardest when the user needs it most.

Task interference by a support application can be mitigated by reducing its perceptual or cognitive demands. One important technique for reducing interaction complexity is context awareness. This is the use of non-explicit user and environmental input, typically acquired through sensors[16, 3, 22]. Context awareness can reduce, or in some cases eliminate, the need for explicit user input for some applications.

However, context awareness can only directly address half of the interaction problem - the part about getting in- 
formation into the application. Getting information out of the application and into the user still presents substantial perceptual and cognitive demands. While context awareness can help a wearable to determine what kind of resources a user currently has available - are they driving a car, or sitting in their office? - it must still present its information somehow, or it will fail to be of use.

Such demands can be lessened by reducing the complexity of information displays and reducing the amount of interpretation required by the user [28, 27]. However, any interaction strategy that requires user perception of computer output will necessarily require the kinds of processing that can interfere with the performance of the real-world tasks. This is unfortunate, because it implies that task interference is an inevitable part of task support. Or is it?

\section{Subliminal Task Support}

Our suggestion is that it is possible to substantially and significantly improve task performance through the use of a wearable subliminal cuing system without the conscious attention or awareness of the user.

We published a pilot study[5] that suggested that this might be possible. In this paper we describe the results of a larger study and compare this to our pilot, with particular emphasis on task interference.

\section{Cognitive Science}

This research has been grounded in cognitive and perceptual psychology. In this section we discuss that context, and in succeeding chapters discuss our own experimental design and results.

The reason we must discuss the primary cognitive and perceptual psychology sources and not an established literature of subliminal HCI is that there isn't one. Aside from our own pilot, we have only been able to locate one other publication of any note about subliminal task support in software. It dealt with desktop HCI and was published in 1991 (F. Layne Wallace, et al. "The Effect of Subliminal Help Presentations on Learning a Text Editor,[26]).

\subsection{Attention}

Contemporary theories of attention began with Broadbent's "Early Selection Theory" in 1958[2]. This model proposed that while all stimuli are analyzed at a physical (nonsemantic) level, only attended stimuli are processed further. "Late-selection" models, such as the one proposed by Deutsch and Deutsch[4], were developed in response to mounting evidence of high-level semantic interpretation of unattended stimuli, such as the ability to accurately shadow a message that jumped back and forth between ears in a dichotic listening task based on semantic content[8].

Modern theories of attention have moved beyond the early- vs. late-selection debate. The evidence now generally supports a compromise "attenuation" theory[25], in which filtering attenuates, but does not prevent, the processing of unattended stimuli. Current research focuses on the finegrained attentional aspects of different perceptual modes and tasks - see [17] for a discussion of contemporary attention research.

\subsection{Unconscious Cognition}

"Unconscious cognition" is essentially the processing of information by the brain in ways that do not require or provoke the awareness that the processing is occurring.

In "A New Look: 3," Greenwald observes that unconscious cognition is solidly established in empirical research, though in a form substantially different from (and simpler than) that put forward by classic psychoanalytic theory[9].

High-level semantic processing of unattended stimuli provides strong evidence for unconscious cognition. Further evidence lies in the phenomenon of automatic processing[20]. Highly practiced skills, such as word reading in literate adults, appear to be automatic and parallel, in that little demand is placed on attention or short-term memory. (Other examples include many aspects of driving, or playing a musical instrument.) Controlled processing is just the opposite - it places demands on attention and short term memory, and is highly serial.

Once a processing task becomes automatic, it may also be inevitable, in that it will always occur when an appropriate stimulus is presented. This is illustrated by the Stroop effect[23], which is a classic: when subjects are shown a color word (for example the word "green") printed in ink of a different color (for example, blue ink), they find it very difficult to say the name for the ink color aloud ("Blue.") rather than reading the word ("Green."). Processing the word as written language is inevitable, even as it interferes with the consciously-selected controlled processing task of naming the color of ink.

\subsection{Subliminal Perception}

Stimuli can be divided into three categories, based on their availability to attention:

1. the consciously-perceived,

2. the perceptible-but-not-attended-to, and

3. the subliminal.

Subliminal stimuli are those which fall below the subject's threshold of awareness. They may be presented for very short duration, at very low intensities, at frequencies outside the range of conscious perception, or hidden behind 
a "mask" of other stimuli[11]. An important aspect of subliminal stimuli is that they cannot be attended to; they are not available for conscious perception. If a person can't consciously perceive something, they cannot choose to direct their attention towards it.

But the fact that such stimuli cannot be attended to does not mean that they do not get processed by the brain in ways that affect subject performance. Deutsch and Deutsch[4] and Treisman[25] observed high-level semantic processing of unattended stimuli. Von Wright et. all[29] and Govier and Pitts[7] found that subjects were capable of discriminating between alternative meanings of unattended stimuli that the subjects were not consciously aware of. Further, Dixon[6] and Groeger[10] have shown that subliminal stimuli may be subject to high-level semantic, memory, and emotional processing, as in the case of "perceptual defense" - the selective inhibition of conscious perception of disturbing or embarrassing material.

\subsection{Subliminal Cuing for Wearable and Mobile Ap- plications}

Marcel[12, 13] used masked visual stimuli to show that subliminal stimuli have semantic-level priming effects. We use a similar procedure to investigate the effect of subliminal visual cues in support of a memory task.

Our operational definition of a cue is a stimulus that we intend to affect the user's performance on a memory task. An overt cue is a cue that can be consciously perceived and reported by the user. A subliminal cue is a cue that cannot be consciously perceived or reported. Both overt and subliminal cues may produce measurable effects on task performance. In another axis, cues may also be correct relative to the task at hand, or they may be miscues that are contextually inappropriate.

In 1991, Wallace showed that subliminal cuing decreased reliance on online help in a desktop-computer text editing task application[26]. In essence, this was a use of subliminal cues in support of a memory task. Subliminal cues were shown to affect the subjects' conscious use of overt cues.

Since Wallace, little work has been done since to explore the use of subliminal cuing in the domain of human computer interaction. This may be because the common use of modern desktop computers, with their high-resolution displays and their users seated on chairs and concentrating upon the screen, provide a very large quantity of available interaction bandwidth. In such a context, there is little need to reduce the attentive demands of the interaction.

As we explained earlier, issues of divided attention are of much greater concern in wearable task-support applications. A common purpose of such applications is memory support, and indeed much effort has already gone into designing wearables (such as CMU's VuMan series) that provide checklist-style memory support[21]. Jennifer Ockerman[15] refers to these systems as task guidance systems, and describes over-reliance problems that can result from their use. For example, an over-reliant user may get caught up in the minutiae of following the list, and lose sight of their original intent. A more effective task-guidance system would support the user's memory of the procedure without interfering with the task, potentially reducing or eliminating over-reliance problems. Subliminal cues might be a good mechanism for providing that.

\subsection{Subliminal Cuing and Attention}

The automatic nature of subliminal perception means that the processing of subliminal cues should not distract the user's attention, nor interrupt any consciously-controlled cognitive processing tasks. Therefore, subliminal cues should have a minimal effect on the user's overall cognitive load.

The rapid, parallel nature of automatic processing and lack of conscious distraction associated with subliminal cuing leads us to believe that the use of a "subliminal channel" for memory support could be of great value in circumstances where overt cues would be distracting, inappropriate, or otherwise undesirable.

\subsection{Miscues and the Stroop Effect}

The existence of Stroop effects demonstrates that automatic cognitive processes can interfere with consciouslycontrolled processes. (As described earlier, the classical example involves presenting a subject with a word like "green" written in blue ink, and asking them to report the ink color[23].) Can inappropriate cues, be they subliminal or consciously perceived, interfere with memory retrieval or other cognitive processing? This is a very important issue for the design of task-support applications. If the application makes a mistake, what will that do to the user's performance?

\section{Subliminal Memory Support Experiment}

In order to investigate the questions raised in the previous section, we conducted a just-in-time memory support experiment employing subliminal visual cues delivered using a head mounted display. This experiment was designed to simple enough to analyze, but close enough to a "real world" task that the applications would be clear. To the best of our knowledge, our work is the first to investigate subliminal cuing as a human-computer interaction technique for wearable computing. 


\section{1. "Social Memory Assistant" Scenario}

We chose as our concept a just-in-time memory support application to improve the user's memory of face/name associations - a common task in social settings, and one many people find difficult. Further, we assume that process by which our memory support application identifies people may be unreliable; a reasonable assumption given the current limitations of face recognition technology.

Our experiment is intended to begin to investigate the feasibility of constructing such a "Social Memory Assistant" application using subliminal visual cuing. In this experiment we use a wearable computer and head-mounted display to deliver just-in-time memory support cues, some of which are wrong. However, careful testing of memory effects is difficult, and we chose to use a desktop computer as a stand-in for the actual social interaction.

By using a desktop computer for the presentation and testing, we were better able to control for non-experimental conditions than would be possible given a more "real world" setting for this task, such as introducing the subject to a room full of people. However, an obvious limitation of this design is that its highly structured nature is not necessarily a good match for the realities of a cocktail party. We hope to address this in future work.

\subsection{Experimental Hypothesis}

We are interested in investigating the effect of an unreliable subliminal memory aid - one that will occasionally (or frequently) mis-identify a situation and provide an incorrect cue. For this reason we are interested in comparing recall performance under three experimental conditions: no cues (unassisted), correctly cued (assisted), and incorrectly cued (mislead).

The inclusion of incorrectly cued recall as an experimental condition is important for two reasons. First, we assume that any real memory support application will have to operate with imperfect information, and errors are inevitable. Our pilot study suggested that consciously perceived incorrect cues can interfere with memory recall, but subliminal cues may not. In any case, it is important to investigate the impact on performance caused by incorrect cues. Second, subject performance on miscued trials provided corroborating evidence for conscious (non-subliminal) perception of cues in our pilot study[5] and helped us design genuinely subliminal cues for this experiment.

We hypothesized that our subjects would do better on the correctly cued memory trials than the uncued trials, i.e. we expected the effect of the correct cues would be positive. We further hypothesized that that subjects would do no better, and perhaps slightly worse, on the miscued trials, i.e. we expected the effect of the miscues to be zero, or slightly negative.

\subsection{Procedure}

Each subject was assigned to a single trial sequence (see below) and oriented. Subjects were informed of the nature of the memorization task and the presence (but not the method, content, or correctness) of subliminal cues. Subjects were instructed to do as well as possible on the memory trials, and to guess rather than leave a trial unanswered.

After orientation and signing the consent form, subjects donned the monocular MicroOptical QVGA clip-on head mounted display (see below) and were instructed on its proper position and orientation. The display was oriented so that it would appear centered in the subject's field of view when they looked at the desktop computer display.

Each subject was given two minutes to memorize twenty-one name-and-face pairs. The name/face pairs were presented as a web-page in a seven-by-three grid. The duration was timed automatically.

The names were chosen at random from a list of historically popular American women's names, and pictures of women were chosen at random from the FERET[18] face database. (Women were chosen for stimuli because there is more variation in women's names than in men's.)

After the memorization period there was a brief pause, during which the subject indicated that they were ready to proceed. Then the memory trials began. In each trial, he subject was shown one face from the memorization set, and attempted to produce (type) the associated name. Each memory trial was ten seconds in length, and the subjects could answer anytime during that period.

During the memory trials, the subject might or might not receive a subliminal cue on a separate head-mounted display, and this cue might or might not be correct (see below). The cues would occur once per second during the ten seconds that the subject had in which to produce the name hence the "just-in-time" nature of the memory support.

After all trials were completed, the subject was given a questionnaire to complete and the protocol was finished.

\subsubsection{Design Overview}

Our experiment was designed for within-subjects analysis, employing three experimental conditions: uncued, correct cues, and incorrect cues. For each of the three conditions, the subject was presented with seven memory trials, resulting in a total of 21 memory trials per subject.

We knew that some names and faces would be more memorable than others. To control for this effect across subjects, we created three sequences of memory trials to balance the use of a particular name and face across the experimental categories. Thus, the total number of subjects in our data analysis, 21 , is also divisible by three. 
The number of subjects equaling the number of faces in the trials is simply a coincidence, and not an important feature of the design. In fact, a total of 28 subjects were run. 4 runs were discarded for protocol violations (such as the experimenter failing to actually put the wearable on the subject). 3 more were discarded so as to balance the number of subjects across sequences (see below); in these cases the first valid runs were used for the analysis.

Because we used a within-subjects design, we are able to examine the effect of the experimental conditions on a particular subject's performance. For example, we can say that a particular subject did two questions better on correctly cued memory trials than uncued memory trials. More importantly, we can analyze this within-subject effect across subjects, allowing us to control for individual differences in memorization skills.

\subsection{Memory Trials}

The trials were divided into three conditions of seven trials each per subject:

Condition "n": uncued trials,

Condition "C"': correct subliminal cuing,

Condition " $\mathrm{X}$ ": misleading subliminal cuing.

In " $n$ " trials, no subliminal cuing occurred. This was the control condition. In " $\mathrm{C}$ " condition trials, correct subliminal cues were provided (see technique described below). In " $\mathrm{X}$ " condition trials, misleading cues were provided using the same technique as the " $C$ " condition trials.

To balance the design, each of the 21 faces was randomly assigned a common name, and then randomly grouped into three groups of seven faces: "I", "II", and "III". We generated three sequences of trials, labeled Sequence 1, Sequence 2, and Sequence 3. In each sequence, the face groups described above were assigned to experimental categories in rotation, i.e.: $\quad$ Sequence $1 \quad n=I, \quad C=I I, \quad X=I I I$.

Sequence $2 \quad n=I I, \quad C=I I I, \quad X=I$.

Sequence $3 \quad n=I I I, \quad C=I, \quad X=I I$.

The result was three balanced sequences of 21 trials each. The order of the trials was randomized and then sorted such that no more than two successive trials of any condition were present in any sequence.

Subjects were assigned a trial sequence on a rotating basis, such that one third of our subjects experienced each trial sequence. In this way, the experiment was balanced so that each face appeared in each condition for a third of our subjects. Further, the different ordering of the sequences provided controls for primacy and recency effects.

\subsection{Equipment}

Memorization and testing were conducted using a conventional desktop display and keyboard. Subliminal cuing was provided by a head-mounted display worn on the subject's own eyeglasses, or blank optical-quality glasses for subjects who did not wear glasses. The head mounted display was worn during the memorization period as well as during the memory trials, but only active during the trials. The subliminal output device was was a MicroOptical QVGA clip-on head-mounted display driven by custom display hardware on the MIThril 1000 wearable computing system.

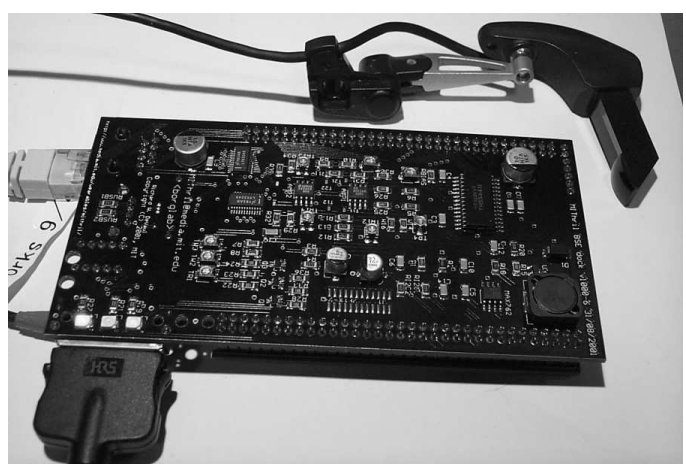

Figure 1. MIThril BSE Dock video driver hardware and MicroOptical QVGA clip-on display

We designed this hardware, shown in Figure 1, to provide complete control of all display parameters, including the timing and sequencing of backlights. This allowed us to precisely control the duration of the subliminal cuing frames (described below) and to adjust this parameter independently of the duration and sequencing of other display information.

The detailed control provided by this video hardware allows us to precisely calibrate the duration and intensity of subliminal cuing frames. We hypothesize that by adjusting these for individual subjects, we may be able to produce even stronger effects in future experiments. In order to allow replication of these experiments by others, the design for the driver hardware and source code for the software is available on the MIThril web site [19].

\subsection{Method of Cuing}

Because we wanted to simulate the conditions of a "real world" subliminal task support application, all subjects were told in advance that the experiment would involve subliminal visual cuing. They were not told anything about the nature of the cues, such as the existence of incorrect cues. 
Cues, in the form of short-duration masked video frames, were displayed on the HMD during the memory trials. The type of cue depended on the condition of the trial, which was unknown to subject and investigator during the running of the experiment.

During each trial the HMD cycled through ten static masking images, one every second. At the transition between images, a 1/180th second cue frame was inserted, resulting in 10 cues per memory trial.

For uncued "n" trials, this frame was blank (black). For correctly cued " $\mathrm{C}$ " trials, the cue frame contained the correct answer (name) spelled out in a large sans-serif font on a black background. For misleadingly cued " $\mathrm{X}$ " trials, the cue fame contained a plausible but incorrect answer - a name associated with a different face in the experiment. Example cue and masking images can be seen in Figure 2
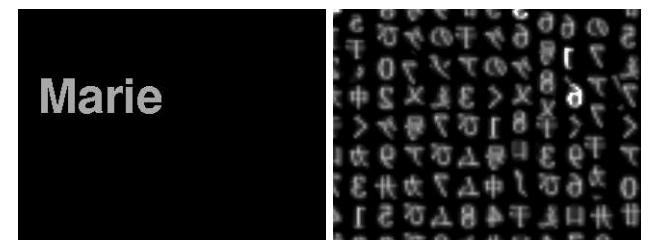

Figure 2. Example Cue and Mask Image

\subsection{Results}

The within-subjects analysis of our 21 subjects' data is summarized in Table 1 and Figure 3. The effect was the difference in performance - that is, the number of correct names produced - between control and experimental conditions for each subject.

The mean effect of correct cuing ("C" trials) was found to be 0.76 , or an improvement factor of 1.47 over the uncued " $n$ " trials. The mean effect of misleading cuing (" $X$ " trials) was found to be 0.43 , or an improvement factor of 1.26 over the uncued trials.

We used a one-tailed $\mathrm{T}$ test to test the hypothesis that the effect of correct cuing ("C" condition) was positive. We found this to be acceptable at $p=0.02$. We used a twotailed test to test whether the hypothesis that the effect of of misleading cuing (" $\mathrm{X}$ " trials) was non-zero; this was borderline significant at $p=0.06$.

\subsubsection{Miscues and Spreading Activation}

As shown in Table 1, the effect of the miscued condition was a borderline-significant increase in performance. While we were not expecting significant task interference, the improvement in response to incorrect cuing was a surprise.

We suggest that an explanation for this counterintuitive effect lies in our choice of miscues. While the name shown in a miscued trial was incorrect for the particular face on the screen at the time, that name did come from the same memorization task, associated with some other face. So the slight increase in performance can be explained by an associative or spreading-activation theory of memory[1].

If our spreading-activation hypothesis is correct, we would not expect to see the same effect if our miscues were completely unrelated to the memorization set. Further study is needed to investigate the effect of different types of miscues.

\begin{tabular}{|r|l|l|l|l|}
\hline cond. & mean & effect & effect $\sigma^{2}$ & Student T $p$ \\
\hline "n" & 1.62 & & & \\
"C" & 2.38 & 0.76 & 2.59 & (one-tail) 0.02 \\
"X" & 2.05 & 0.43 & 0.96 & (two-tail) 0.06 \\
\hline
\end{tabular}

Table 1. Effect of Subliminal Cuing

\begin{tabular}{|r|l|l|}
\hline S mean & O mean & O mean / S mean \\
\hline 0.25 & 2.33 & 9.32 \\
\hline
\end{tabular}

Table 2. Subliminal vs. Overt Miscues: Average Number of Miscued Answers Chosen
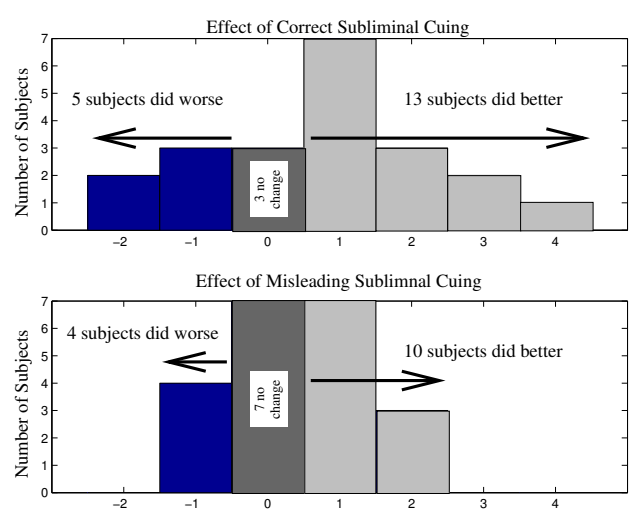

\section{Figure 3. Histograms comparing the effect of correct ("C") and incorrect ("X") cues.}

\subsection{Miscues and Task Interference}

Some subjects in our pilot study[5] were able to consciously perceive the cues. (The pilot's masking protocol was less effective than that of this experiment.) Not only were these subjects able to report the nature of the cues they were being given, we also had corroborating evidence in the form of the performance of these subjects on " $\mathrm{X}$ " 
condition trials. In every case, a pilot study subject who reported being able to read the cues showed at least one incident of changing their answer on an " $\mathrm{X}$ " trial from a correctly remembered answer to the incorrect cued one. (Since all keystrokes were recorded, the data showed the subject typing in the correct name, then deleting it and typing in the incorrect name shown by the cue.) Further, subjects who were able to consciously perceive cues were nearly ten times as likely to choose the incorrect cued answer on " $X$ " trials than subjects who could not, as shown in Figure 2. Post hoc analysis of the data suggest that this is a highly significant effect, although it was not a well-defined factor and we are reluctant to make claims of statistical validity. In future work we will investigate the effect of overt miscues more carefully.

By contrast, subjects in the pilot study who did not report being able to perceive the cues were no more likely to guess the incorrectly cued name on an " $X$ " trial than any other name used in the study, and showed no evidence of substituting an incorrectly cued answer for a correctly remembered one.

The difference in performance on the " $\mathrm{X}$ " condition trials between subjects who could and could not consciously perceive the cues in our pilot study suggests an important difference between subliminal and overt cuing: Incorrect subliminal cues appear not to interfere with memory recall, but incorrect overt cues do.

If this result is supported by further research, it indicates a major strength of subliminal cuing for task support. Applications that use overt cuing are likely to cause the user to make the same mistakes that the program does. But applications that use subliminal cuing are unlikely to propagate their mistakes into their users' performance.

\section{Conclusions}

Our subjects did significantly better on the "C" trials than the "n" trials, showing that wearable subliminal visual cuing is effective as a just-in-time memory retrieval aid. The misleading cues in our " $\mathrm{X}$ " condition did not interfere with correct recall; indeed, a small (though borderline significant) improvement was observed, as discussed in Section 4.7.1 above. These results support our experimental hypothesis about correct subliminal cues. While they do not support our original hypothesis about incorrect subliminal cues, the observed results actually make an even better case for the usefulness of subliminal cuing than we had expected.

At this point we have good reason to believe that subliminal cuing can be an effective, low-attention alternative to overt cuing for memory support, and can provide more resilience for the user against mistakes made by the application.

Our results regarding miscues are particularly important.
Effective memory support requires situation-appropriate information. However, perfect knowledge of the user's situation may be difficult or impossible to obtain for many realworld applications. Our research suggests that delivering overt cues based on imperfect information risks distracting and misleading the user. However, it appears that at least some types of incorrect subliminal cues are benign, and under some circumstances may even support correct performance.

If these results are supported by future research, it will indicate that subliminal cues could be used effectively and with greater safety than overt cues in applications that must rely on less-than-perfect knowledge of the user's context. This could make subliminal cuing quite attractive for realworld wearable task-support applications.

\subsection{Limitations}

There are a number of limitations in this work. First, only one type of miscue was included in our study. Although our particular miscues appear helpful, it is possible that other types of subliminal miscues might result in performance degradation. Second, our data regarding the negative effects of overt cuing was not the result of a careful experiment to test this phenomenon. The effects of incorrect overt cuing on memory retrieval still need direct study. Third, our study used a highly controlled memorization and testing environment. In order to make strong claims about the effectiveness of subliminal cuing for real-world applications, we must demonstrate the effect under more naturalistic conditions.

\section{Future Experimental Work}

One of the most interesting aspects of our results thus far is the apparent lack of task interference produced by incorrect subliminal cues, as opposed to the obvious task interference effect (in the form of changed answers) we observed in our pilot study among subjects who could consciously perceive the cues. This startling finding provokes some new and interesting ideas to investigate.

\subsection{Recall Interference and Overt Cuing}

We hope to investigate the presentation of two overtly cued experimental conditions: correct overt cues and misleading overt cues. While some data of this sort was incidentally available from our pilot, a study designed to investigate it more directly will allow us to address questions about subjects' ability to perform the meta-memory task of discriminating a correct from an incorrect overt cue. We will also investigate the effect of subject expectations: do subjects who are explicitly warned that incorrect cues may 
be presented behave differently from those who are not thus prepared?

This study will also allow us to investigate the effects of different types of miscues, both overt and subliminal. For example, we might compare the relative effects of incorrect names chosen from the memory set, names outside the memorization set, randomly chosen nouns, and strings of nonsense syllables.

\subsection{Ambulatory "Social Assistant"}

Another important study is replicating our "Social Memory Assistant" in an ambulatory context, where the subject is introduced to a previously unfamiliar group of people and asked to learn their names. Using active tags we can provide the wearable with near-perfect knowledge of the correct responses, and investigate the effects of correct and misleading cuing in a more naturalistic social environment.

\section{References}

[1] J. R. Anderson. A spreading activation theory of memory. Journal of Verbal Learning and Verbal Behavior, 1983.

[2] D. E. Broadbent. Perception and Communication. Pergamon Press, London, 1958.

[3] P. J. Brown, J. D. Bovey, and X. Chen. Context-aware applications: From the laboratory to the marketplace. IEEE Personal Comunications, 4(5):58-64, October 1997.

[4] J. A. Deutsch and D. Deutsch. Comments on 'selective attention: perception or response?'. Quarterly Journal of Experimental Psychology, 19:362-363, 1967.

[5] R. DeVaul and S. Pentland. Toward the zero attention interface. In Digest of Papers. 6th International Symposium on Wearable Computers, pages 141-142. IEEE Computer Society, October 2002.

[6] N. F. Dixon. Perceptual and other related defenses. Polish Psychological Bulletin, 21(4):319-330, 1990.

[7] E. Govier and M. Pitts. The contextual disambiguation of a polysemous word in an unattended message. British Journal of Psychology, 73(4):537-545, November 1982.

[8] J. A. Gray and A. A. I. Wedderburn. Grouping strategies with simultaneous stimuli. Quarterly Journal of Experimental Psychology, 12:180-184, 1960.

[9] A. G. Greenwald. New look 3: Unconscious cognition reclaimed. American Psychologist, 47(6):766-779, 1992.

[10] J. A. Groeger. Qualitatively different effects of undetected and unidentified auditory primes. Quarterly Journal of Experimental Psychology, 40(2):323-329, May 1988.

[11] D. Groome, H. Dewart, A. Esgate, K. Gurney, R. Kemp, and N. Towell. An Introduction to Cognitive Psychology: Processes and Disorders, chapter 2.12. Psychology Press Ltd, 1999.

[12] A. J. Marcel. Conscious and unconscious perception: An approach to the relations between phenomenal experience and perceptual processes. Cognitive Psychology, 15(2):238300, April 1983.
[13] A. J. Marcel. Conscious and unconscious perception: Experiments on visual masking and word recognition. Cognitive Psychology, 15(2):197-237, April 1983.

[14] L. Najjar, C. Thompson, and J. Ockerman. A wearable computer for quality assurance inspectors in a food processing plant. In IEEE Intl. Symp. on Wearable Computers. IEEE Computer Society, 1997.

[15] J. J. Ockerman. Task guidance and procedure context: Aiding workers in appropriate procedure following. $\mathrm{PhD}$ thesis, Georgia Institute of Technology, 2000.

[16] J. Pascoe. Adding generic contextual capabilities to wearable computers. In Digest of Papers. Second International Symposium on Wearable Computers, pages 92-99. IEEE, October 1998.

[17] H. Pashler, editor. Attention. Psychology Press, 1998.

[18] P. J. Phillips. The feret database and evaluation procedure for face-recognition algorithms. Image and Vision Computing Journal, 16(5):295-306, 1998.

[19] The MIThril project website. http://www.media.mit.edu/wearables/mithril, 2001.

[20] R. M. Shiffrin and W. Schneider. Controlled and automatic human information processing: II. perceptual learning, automatic attending, and a general theory. Psychological Review, 84:127-190, 1977.

[21] A. Smailagic and D. Siewiorek. The CMU mobile computers: A new generation of computer systems. In COMPCON '94. IEEE Computer Society Press, February 1994.

[22] T. Starner, B. Schiele, and A. Pentland. Visual context awareness in wearable computing. In Digest of Papers. Second International Symposium on Wearable Computers, pages 50-57. IEEE Computer Society, October 1998.

[23] J. R. Stroop. Studies of interference in serial verbal reactions. Journal of Experimental Psychology, 18:643-662, 1935.

[24] L. Sydiinheimo, M. Salmimaa, J. Vanhala, and M. Kivikoski. Wearable and ubiquitous computer aided service, maintenance and overhaul. IEEE International Conference on Communications, 3(99CH36311):2012-17, 1999. Inst. of Electron., Tampere Univ. of Technol., Finland.

[25] A. M. Treisman. Verbal cues, language and meaning in selective attention. American Journal of Psychology, 77:206219, 1964.

[26] F. L. Wallace, J. M. Flanery, and G. A. Knezek. The effect of subliminal help presentations on learning a text editor. Information Processing and Management, 27(2/3):211-218, 1991.

[27] C. Wickens. Situation awareness: impact of automation and display technology. In NATO AGARD Aerospace Medical Panel Symposium on Situation Awareness, AGARD CP-575, Neuilly-Sur-Seine, France, 1996.

[28] C. Wickens and T. Prevett. Exploring the dimensions of egocentricity in aircraft navigation displays: influences on local guidance and global situation awareness. J. of Experimental Psychology: Applied, 1(2):110-135, 1995.

[29] J. M. V. Wright, K. Anderson, and U. Stenman. Generalisation of conditioned GSRs in dichotic listening. In P. M. A. Rabbitt and S. Dornic, editors, Attention and Performance. Academic Press, London, 1985. 